\title{
Desarrollo de una Identidad \\ con Tendencia a la Vergüenza \\ Una mirada desde la Teoria de E. Erikson y las \\ Interacciones Familiares
}

\section{TaniaDonoso Niemeyer ${ }^{1}$}

\begin{abstract}
Resumen
Este artículo intenta relacionar la resolución de los estadios del desarrollo psicosocial planteados por E. Erikson: Autonomía vs. Vergüenza y Duda Iniciativa vs. Culpa, con el desarrollo de una identidad saludable o de una identidad con tendencia a la vergüenza (concepto desarrollado por Harper \& Hoopes, 1990).

En seguida se discuten algunos procesos de interacción familiar que facilitan la estructuración de una identidad con tendencia a la vergüenza en una persona, como también características del funcionamiento familiar que permiten la conformación de una identidad saludable.

Finalmente se analiza la función que cumpliria el proceso de diferenciación personal en la posibilidad de cambio desde una identidad con tendencia a la vergüenza hacia una identidad saludable.
\end{abstract}

Palabras claves: Identidad, Vergüenza, Culpa, Interacción Familiar

\begin{abstract}
This work is an attempt to relate the resolution of the E. Erikson's stages of psychosocial development: Autonomy versus Shame and Doubt and Initiative versus Guilt, with the development of a Healthy Identity or a Shame-Prone Identity (a concept developed by Harper \& Hoopes, 1990).

Some family interactional processes that facilitate the development of a Shame-Prone Identity in human beings, as well as some elements of family functioning that permit the development of a Healthy Identity are discussed next.

Finally the function of the Differentiation of Self process in the developmental change from a ShameProne Identity to a Healthy Identity is analysed.
\end{abstract}

Keywords: Identity, Shame, Guilt, Family Interactions

\section{DesarrolloPsicosocial Temprano}

Los infantes desde el nacimiento son capaces de experimentar interés, angustia, disgusto. A los pocos meses expresan alegria, rabia, sorpresa, timidez y miedo. El aprendizaje acerca de sí mismo (conciencia de sí mismo, autoimagen y autovaloración) comienza tempranamente en las relaciones que establece con otras personas pertenecientes básicamente a su núcleo familiar. Emociones complejas como empatia, celos, vergüenza, culpabilidad y orgullo surgen alrededor de los 18 meses cuando ya 
se ha desarrollado la autoconciencia y el autoreconocimiento (Lewis, 1990). Lo anterior, junto con el avance en el desarrollo cognitivo, capacita a los niños a pensar sobre sus propios sentimientos, desarrollar sentimientos de empatía, siendo capaces poco a poco de colocarse en el lugar de otra persona (Papalia, 1997).

Erik Erikson (1974) plantea que el desarrollo de la personalidad es un proceso de búsqueda de un sentido de identidad que comprende toda la vida. En él se deben resolver los conflictos creados por fuerzas biológicas (fortalezas y debilidades biológicas personales), psicológicas (historia de desarro1lo, experiencias familiares tempranas, circunstancias de vida únicas y cómo ha resuelto las crisis anteriores del desarrollo) y sociales, culturales e históricas que operan en la vida particular de cada persona.

Erikson afirma que este proceso ocurre en ocho estadios, cada uno de los cuales corresponde a la época en que una capacidad particular aparece por primera vez. Cada estadio involucra una crisis psicosocial particular y un período crucial de aumento de vulnerabilidad, que cada individuo viviendo en una determinada sociedad, debe resolver para lograr una identidad adulta. De este modo, la solución eficaz de los conflictos correspondientes a cada crisis provoca un movimiento ascendente de madurez.

La tarea evolutiva del período del nacimiento hasta los 18 meses, es el logro de la Confianza Básica versus la Desconfianza Básica, que permite al niño confiar en sí mismo y en su medio para explorarlo sin temor. El convenio "estar de acuerdo con la madre" que caracteriza esta etapa debe quebrantarse en la medida que el niño desarrolla la autoconciencia y sustituye cada vez más el juicio de los adultos por el propio.

Los niños entre 18 meses a 3 años, normalmente experimentan la crisis de Autonomía vs. Vergüenzay Duda, en la cual necesitan lograr un equilibrio entre la autodeterminación y autocontrol, y el control externo. Necesitan aprender lo que pueden y deben hacer, lo que es seguro hacer, y la clase de guia que aún necesitan de sus padres. Surge la virtud de la voluntad, aprender a hacer sus propias elecciones y decisiones, a ejercitar la autorestricción y seguir sus propios intereses. Para lograr la autonomía necesitan suficiente control y guía por parte de los adultos significativos que lo rodean.

La tercera crisis psicosocial, Iniciativa us. Culpabilidad, es enfrentada por los niños entre los 3 y 6 años de edad. Durante este período, los niños deben lograr un equilibrio entre el deseo de perseguir sus propios objetivos y las restricciones morales de su cultura que les impiden llevarlos a cabo. Desarrollan la virtud del propósito, el valor del prever y perseguir metas, sin inhibirse por la culpa o el miedo al castigo. En la medida que los padres permitan a los niños a hacer las cosas a su modo respaldándolos con guía y límites firmes, favorecerán su sentido de iniciativa, sin inhibirlos ni hacerlos sentir culpables.

\section{Desarrollodela Identidad}

El concepto de identidad se refiere a poseer un sentido internalizado, coherente y estable de quién es uno, qué se desea, y qué se cree y valora (Bukatko, 1995). Erik Erikson plantea que la identidad resulta de la integración de una variedad de identificaciones pasadas de la infancia con identificaciones presentes tanto a nivel consciente como inconsciente. Este proceso, si bien se inicia en la infancia desde el comienzo de la diferenciación del sí mismo como un ser separado de otro, se construye principalmente durante la adolescencia y adultez temprana, terminando con la integración en la sociedad y por último con la aceptación de la propia vida y luego de la muerte al término de la vida.

Sepúlveda (1996), reconceptualiza las ideas Eriksonianas señalando que la identidad "es un procesoen elque seincorporan las partes más omenos disgregadas del simismoen unaunidad flexible yefectiva. Esunaestructura yorganizaciónquecambiaconlaedad y la experiencia. La emergencia de un sentido de unicidadeindividualidadseadquiere del conocimiento deque el simismo es diferente deotros, y tiene sus propias caracteristicas, pero que a la vez comparte ciertas caracte- 
risticas comunes con otros". También destaca la relevancia de las relaciones interpersonales significativas en la estructuración de la identidad: "la relación interpersonal es clave en el desarrollo de laidentidad personal. La diferenciación del self selogra a través de una sintesis delas identificaciones previas con figuras significativas".

De acuerdo con E. Erikson, el logro de la aceptación y confianza en los demás en una etapa temprana, el haber sido incentivado a explorar intereses y deseos y haber logrado adquirir sentimientos de competencia y habilidad, son elementos fundamentales en la conformación de una identidad saludable (Bukatko, 1995). Por otro lado, Harper y Hoopes (1990), plantean que el desarrollar la autoafirmación es fundamental en la estructuración de una identidad saludable. Con este término se refieren a un proceso interactivo con otras personas, donde el individuo experimenta el sentimiento que su propia existencia es confirmada y reconocida por los demás.

\section{Vergülenzayculpa}

Harper \& Hoopes (1990) diferencian claramente los conceptos vergüenza y culpa. La vergüenza corresponde a una emoción en respuesta a una evaluación negativa del propio self, en cambio la culpa apunta a una evaluación negativa del comportamiento.

Cuando la vergüenza es vivida por un período transitorio cumple funciones adaptativas en la familia (Bradshaw, 1988; Zzard, 1977; Kaufman, 1989). Permite aprender a conducirse de acuerdo a los estándares conductuales de la identidad del grupo de pertenencia y a reconocer los limites personales yde los demás. (Bradshaw, 1988; Friesen, 1979). Este mecanismo previene el desarrollo de una autoimagen sobrevalorada $\mathrm{o}$ arrogante, $\mathrm{y}$ facilita que la persona tenga una imagen de sí mismo como "buena persona, si bien no perfecta", pero capaz de cambiar las conductas que no le gustan.

Harper \& Hoopes (1990) plantean que la culpa concebida como una evaluación de la conductay no del sí mismo cumple la función de ayudar a preservar el orden, a mantener la mutua dependencia y a establecer significados y valores en las relaciones humanas, todo lo cual favorece un desarrollo saludable.

De acuerdo a lo planteado por Erik Erikson, la vergüenza tiene una aparición evolutivamente anterior a la culpa. La vergüenza surge en el niño pequeño como oposición al logro de un sentido de autonomía y autodeterminación, en tanto que la culpa surge en la etapa siguiente en la medida que el niño no logra equilibrar sus deseos y objetivos, con las restricciones sociales y morales de su cultura.

Considerando este punto de vista, la vergüenza perturbaria más significativamente el sí mismo, puesto que constituye una amenaza para la diferenciación y logro de un sentido de unicidad. La culpa en cambio, no atenta tan profundamente contra la imagen del self, sino que castiga a modo de un severo superyo las conductas que la persona ve en si misma como sancionables socialmente. Ambas emociones, vergüenza y culpa al ser experimentadas en forma crónica impiden al sujeto conectarse con sus propias y legitimas necesidades, y actuar en el mundo libremente.

La vergüenza constituye una experiencia emocional de humillación dolorosa que involucra un sentimiento de inferioridad, desazón, de ser insuficiente como individuo, donde la persona desea desaparecer, ser otra, borrar el presente y volver atrás para deshacer lo que es vergonzoso (Fossum \& Mason, 1986; Kaufman, 1989; Tompkins, 1987).

Por otro lado, Miller (1981) señala que algunas personas no viven la culpa como una emoción pasajera donde se evalúa una conducta específica, sino que la experimentan de un modo intenso, excesivo y crónico, utilizándola para avergonzarse aún más de si mismo. Estas personas, denominadas "contendencia a la vergüenza", interpretan frecuentemente los incidentes de sus vidas como una confirmación de cuán poco valen, cuán malos son, cuán poco queribles e incapaces de amar y dar a los demás son. Creen ser "malos" independientemente de lo 
que hagan o de lo que otras personas piensen respecto a ellos, e incluso desconfirman las evaluaciones positivas que los demás realizan de ellos. Suelen tener dificultad para integrar sus sentimientos con lo que piensan y tienen poca conciencia de muchas de sus emociones. Escinden los sentimientos como partes malas de sí, evitando su escondido y perdido verdadero self hasta que una depresión $\mathrm{u}$ otro desorden emocional o mental los lleva a confrontarse a sí mismos. En sus relaciones con otras personas tienden a jugar el papel de víctimas o victimarios, o alternar entre estos dos roles.

Harper \& Hoopes (1990) plantean que una persona puede sin problemas una identidad saludable cuando la vergüenza es experimentada como una emoción transitoria, sin embargo cuando es vivida como un sentimiento permanente va estructurando una identidad con tendencia a la vergüenza, que correspondería identidad negativa, una forma de difusión de identidad puesto que la persona no es capaz de integrar sus necesidades infantiles de aprobación con una perspectiva presente de vida.

\section{PatronesdeInteracción Familiar favorecedoras del desarrollode una IdentidadSaludable}

Epstein, Bishop \& Baldwin (1982) plantean que las familias funcionales proporcionan una afirmación positiva a todos sus miembros, permitiendo que cada uno se sienta querido y valorado. El concepto afirmación alude al "proceso interactivo donde una persona experimenta que la propia existencia está confirmada y reconocida por los demás y por el ambiente". Harper \& Hoopes (1990) enfatizan que la forma en que los padres atienden sus propias necesidades y las de sus hijos determina en gran medida si los niños experimentan la culpa y la vergüenza en forma más permanentemente o transitoria, desarrollando una identidad con tendencia a la vergüenza o no. La conformación de una autoimagen positiva y una identidad saludable en una persona (particularmente en un niño en sus relaciones con sus padres) estará favorecida en la medida que en sus relaciones familiares pueda experimentar los siguientes elementos interactivos:

1. Intimidad

2. Dependencia

3. Contar con el otro

La intimidad es entendida como el sentimiento entre una o más personas que tienen cercanía emocional mutuay que comparten experiencias que se reconocen entre ellos como legitimas en las áreas de la emoción, intelecto, relaciones sociales, recreación, contacto fisico y afecto. Cuando existe intimidad en una familia, cualquier miembro de ésta puede expresar todos sus sentimientos con la confianza que los demás lo seguirán queriendo y no lo abandonarán o rechazarán por expresar sus emociones. Esto permite que disminuyan sus defensas y estimula el desarrollo de la habilidad para escuchar lo que el otro está intentando comunicar. Algunos indicadores de la calidad de la intimidad que se pueden observar en las familias son: proximidad, espacio y capacidad de iniciar y/o aceptar contacto fisico con otra persona.

La dependencia es un proceso iniciado por los padres que permite a los niños pequeños ser dependientes de ellos en beneficio de su sustento fisico, necesidades emocionales y de la adquisición de un sentido de orden, de valores y creencias acerca del mundo. La dependencia mutua permite atender las necesidades de otros miembros de la familia y provee seguridad para todos sus integrantes. Goodrich (1984) enfatiza la necesidad de dependencia en la niñez temprana para poder posteriormente durante la adolescencia desarrollar una identidad saludable. Cuando los padres no aceptan las necesidades de dependencia de los hijos, ocurre que éstos si bien logran independizarse de sus progenitores, no logran una individuación satisfactoria y se vuelven distantes, se aíslan, desconfian de los demás, de su ambiente e incluso de sus propias habilidades.

El contar con el otro corresponde a un proceso en el cual cada miembro de la familia se siente obligados a responder por sus conductas hacia si mis- 
mos y hacia los otros miembros de acuerdo a ciertos valoresy estándares (Semin, 1983; Sholter, 1984). El hecho que "tu puedas contar conmigo y yo pueda contar contigo" implica sentirse responsable del comportamiento de uno respecto a la otra persona, es decir sentir la obligación moral de ayudary atender las necesidades del otro, y viceversa, esperar recibir eso mismo del otro.

De acuerdo a Harper \& Hoopes (1990), los niños en las familias donde están presentes estas tres dimensiones (intimidad, dependencia y contar con el otro) fácilmente aprenden a:

1. Controlar sus vidas haciendo cambios cuando ellos lo eligen.

2. Ser adultos capaces de amary contener.

3. Aceptar las equivocaciones como corregibles y no como afirmaciones que son malos.

4. Saber que son queridos y que sus padres están alli para ellos.

5. Aceptar que es posible cambiar actitudesy conductas como una forma normaly deseable de adaptarse a los requerimientos de la vida en familia.

6. Tomar riesgos sin temor de cometer errores, ya que éstos son vistos como un modo de aprender.

7. Reconocer el mundo como un lugar en el cual por lo general se puede confiar.

8. Confiary pedir ayuda a otras personas.

9. Perdonar las equivocaciones en otros y ser más aceptador de los propios sentimientos y actitudes.

10. Aceptar un amplio espectro de experiencias emocionales como deseables y no amenazantes.

11. Apreciary buscar integrar las habilidades emocionales y racionales.

12. Desarrollar un sentido personal de ética y espiritualidad.

13. Establecer limites apropiados en las relaciones interpersonales.

14. Diferenciar el contexto de los sentimientos per- sonales, creencias y cogniciones para regular el establecimiento de limites apropiados fisicosy de afecto.

15. Respetar reglas implícitas de comunicación, de las personas y las cosas dentro y fuera de la familia.

16. Preservar la identidad única familiar.

Se han destacado una algunas interacciones familiares que favorecen el desarrollo de una identidad saludable como que:

1. En la familia se cree un contexto para que cada persona pueda ser un individuo separado, único y valorado (Kerr \& Bowen, 1988).

2. Los errores y equivocaciones de los miembros de la familia sean vistos como incidentes corregibles más que oportunidades para rotular a los niños o padres como "malos" (Zuk \& Zuk, 1987).

3. Los niños aprendan que son queridos, valorados, apoyados y contenidos emocionalmente por sus padres (Olson et al., 1983).

4. Exista potencialmente en la familia una o más relaciones intimas garantizadas incondicionalmente (Olson, Srenkli \& Russell, (1979).

5. Los valores, estándares y expectativas, estén claramente identificados e incorporados en la conducta de los padres y otros adultos significativos (Schulman \& Melker, 1985).

6. Los valores, estándares y expectativas se señalen de modo consistente por uno o ambos padres, de manera que favorezcan experiencias de culpa más que sentimientos de vergüenza en los niños (Lindsay-Hartz, 1984).

\section{PatronesdeInteracción familiar Contribuyentes al desarrollode una identidad con tendencia ala Verguienza}

Las personas con tendencia a la vergüenzageneralmente pertenecen a familias de origen donde circula la tendencia a la vergüenza en varios de sus miem- 
bros. Se han descrito modos de interacción familiar que se estima que inciden en el desarrollo de una identidad con tendencia a la vergüenza, siendo su severidad dependiente de la intensidad y frecuencia en que se presenten los factores interactivos favorecedores del desarrollo de una identidad de este tipo. En estas familias se suele encontrar lo siguiente:

1. Interacciones donde los padres tratan a los otros miembros de la familia como extensiones de sí mismos y a menudo tratan a los niños como posesiones más que como personas. La formación de identidades separadas y únicas con sus propias necesidades personales son vistas como amenazantes para el sistema (Kerr \&Bowen, 1988).

2. Los errores y equivocaciones establecen un escenario para ser desvalorizado y humillado por los otros miembros de la familia. Se personaliza un ataque y el individuo que cometa una equivocación experimenta un sentimiento de "ser malo"(Stierlin, 1974).

3. Los niños se cuestionan si son amados o no. Sienten tener poco valor para los otros miembros de la familia y no esperan apoyo de parte de ellos (Naiditch, 1987).

4. Los niños criados en este medio familiar donde circula la vergüenza tendrán identidades con tendencia a la vergüenza. Sin embargo, la intensidad y el modo que ellos expresan su tendencia a la vergüenza variará de acuerdo a las posiciones sistémicas de los padres y los hijos (Hoopes \& Harper, 1987).

5. Cualquier sentimiento de intimidad en las relaciones familiares es amenazante y crea ansiedad en los padres y en otros miembros de la familia (Feldman, 1979).

6. Los padres u otros adultos significativos no tienen claramente identificados ni incorporados como propios en su conducta sus valores, estándares y expectativas. Además no se señalan consistentemente y cuando se indican, se hace en forma dura, severay acompañados de fuerza fisicay/o con una intensidad de la emo- ción que es inapropiada para la situación. (Schulman \& Melker, 1985; Harpery Hoopes, 1990).

7. Los niños se sienten inseguros y aprenden a que no pueden contar con los demás para satisfacer sus necesidades personales puesto que el medio familiar se visualiza como un lugar poco predecible (Carnes, 1984).

8. Los padres imponen las reglas familiares de una manera que le enseñan a los hijos a tener miedo del poder y de la autoridad. Las necesidades de dependencia son negadas por la inhabilidad de los otros miembros de la familia para aceptar que alguien dependa de ellos y los padres generalmente esperan que los hijos llenen sus propias necesidades de dependencia no satisfechas (Nethason, 1987).

Harper y Hoopes (1990) enfatizan que el desarrollo de una identidad con tendencia a la vergüenza en un hijo involucra un proceso psicológico en el cual el niño internaliza actitudes parentales que habitualmente resumen una acumulación de vergüenza experimentada en su propia familia de origen. Destacan la relevancia que durante las experiencias infantiles tempranas los padres $y / u$ otros adultos significativos manifiesten actitudes de:

a) incomodidad y/o rabia ante la expresión de necesidades normales de los hijos como las necesidades de dependencia o de intimar.

b) reaccionen en forma negativa e intensa frente a ciertas conductas del niño.

c) manifiesten mensajes verbales y/o conductas que conllevan mensajes no verbales que al ser leídos por los niños los llenan de sentimientos de poco valer, de no ser aceptados y de humillación.

Los niños habitualmente tienen dificultad para discriminar las emociones de rabia, furia, tristeza 0 miedo presentes en sus padres. Cuando éstos emociones están presentes tienden a significarlas proyectivamente como que "ellos son malos y deben eliminar las partes malas de sí mismos" (Miller, 1981). En lo más profundo de su existencia creen que en cualquier contexto donde estén los demás 
piensan que ellos "son malos", porque ellos mismos creen eso, hasta el punto de negar o descalificar a cualquier persona que les ofrezca una visión diferente a la que ellos tienen de sí mismos. De esta manera los niños pierden el contacto con sus emociones y con su verdadero self, y más tarde como adultos lo proyectarán en otros, sin lograr un buen desarrollo de su individuación.

Acontinuación se analizan modos de interacción familiar en etapas tempranas de la vida que inciden en el desarrollo de una identidad con tendencia a la vergüenza. Destaco el hecho que ocurran en el periodo de la vida entre los 2 y 6 años debido a que corresponde al momento en que los niños deben resolver las crisis psicosociales de Autonomía vs. Vergüenzae Iniciativa us. Culpa propias de la edad, en beneficio del desarrollo de un sentimiento de autonomía e iniciativa para explorar su mundo.

Siguiendo los postulados de la psicología del desarrollo, la manera cómo resuelvan las crisis $A u$ tonomía vs. Vergüenza e Iniciativa vs. Culpa influirá no solo en el desarrollo psicosocial puntual de la respectiva edad, sino también posteriormente en el proceso de conformación de la identidad en dirección hacia un modo de funcionamiento saludable o favorecedor de una estructuración de identidad negativa con tendencia a la vergüenza.

Se enfatiza particularmente la interacción madre-hijo puesto que es con la figura materna con quién habitualmente se establece el vinculo temprano más permanente y determinante en el desarrollo afectivo de los niños. Sin embargo el niño será también será afectado si ocurren este tipo de interacciones con cualquier miembro significativo de la familia. Incluso más adelante en el desarrollo otras figuras de autoridad como profesores y pares también cobran importancia en el proceso de desarrollo de la autoafirmación en una persona. Harper \& Hoopes (1990) afirman que las características de los patrones de interacción familiar en la niñez temprana proveen un marco de referencia y filtran como lentes el modo en que los individuos adquieren afirmación personal en sus relaciones con otros adultos o pares fuera del ambiente familiar.

\section{Sobreexigenciasfamiliares.}

Las familias donde la madre u otros miembros significativos adultos son demasiado exigentes y críticos consigo mismos, habitualmente también lo son con sus hijos y depositan expectativas demasiado altas en ellos. Estos padres tienden a sobreexigir al hijo y a validarlo a través de una evaluación constante de sus desempeños. Incluso las expresiones afectivas hacia el niño están estrechamente relacionadas con que el pequeño se comporte y tenga los logros que estos adultos esperan de él.

Puesto que se trata de una sobreexigencia, es decir más allá de lo que el niño puede dar en un determinado momento de su desarrollo, nunca alcanza a ser lo suficientemente eficaz nialcanzar los estándares planteados para cumplir las demandas esperadas por los adultos. Esta situación le genera sentimientos de incapacidad, baja autoestima, e incluso vergüenza y culpa por ser incapaz de responder a las demandas de los adultos afectivamente significativos de su vida.

Con el fin de recibir refuerzo por su conducta el niño aprende a comportarse del modo que él percibe que le agrada a sus padres y adultos significativos. De esta forma, poco a poco va perdiendo su espontaneidad y el sentir placer en conductas que podrian agradarle pero que son distintas a las supuestamente esperadas de él por las figuras significativas de su familia.

\section{Contradicción entre los mensajes verbales y noverbales.}

Algunas familias muestran un alto grado de contradicción entre el nivel comunicacional verbaly el no verbal. Las exigencias y expectativas parentales no se explicitan e incluso los mensajes verbales discrepan de los mensajes no verbales emitidos. Sin embargo, los niños son altamente perceptivos de los códigos comunicacionales no verbales y se ven envueltos entre dos mensajes contradictorios que generan una situación doble-vinculante. Haga lo 
que haga, el niño no llegará a ser o hacer lo que esperan de él, puesto que es imposible por la contradicción solicitada. Entonces se sentirá incapaz, desvalorizado, culpable o avergonzado por no ser capaz de responderle a sus seres significativos.

Aún más, en esta interacción sus sentimientos internos desvalorizados se ven confirmados por el hecho que psicológicamente el nivel no verbalanalógico tiene un mayor peso a nivel inconsciente que el nivel verbal-digital. Watzlawick (1989) hace una clara referencia de esta idea señalando: "puesto quelacomunicación se centraen aspectos relacionales comprobamosqueellenguaje digital carece casiporcompleto de significado".

\section{Bajo nivel de empatia ycontención materna.}

El nivel de empatía que una madre haya desarrollado en el transcurso de su vida se relaciona estrechamente con su capacidad maternal para detectar las necesidades de su hijo en forma efectiva y oportuna. Si la madre tiene una baja capacidad de empatía y un alto nivel de egocentrismo, la relación madre-hijo probablemente estará más centrada en sus necesidades maternales que en las necesidades y emociones del hijo. En el desempeño de su rol maternal le será dificil anticiparse y darse cuenta de los momentos en que el niño necesita apoyo y contención, como también acercarse al mundo del niño esperando en cambio que el hijo sea quien se acerque a su mundo adulto en demanda de sus necesidades.

El hijo, quien necesita mantener el vínculo con la figura materna, aprenderá en las comunicaciones con ella a buscar el modo de satisfacer los deseos de ella. No tendrá el aprendizaje suficiente para saber contactarse con sus propias necesidades o de buscar el reconocimiento de sus propios intereses en la madre. Entonces cuando aparezcan sus necesidades sentirá culpa o las evaluará como "malas" por el sólo hecho de existir.

\section{Capacidaddeescucharselectiva hacia las necesidades del hijo.}

Distintas personas tienen capacidades diferentes para escuchar. Algunos escuchan una gran diversidad de mensajes y otros sólo lo que "quieren" o "pueden" oír. Algunos padres que tienen una capacidad bastante disminuída para escuchar efectivamente lo que el niño quiere decir. Escuchan sólo una parte del mensaje llegando incluso a creer que están escuchando plenamente al hijo, pero en realidad niegan la parte que no fue escuchada o entendida puesto que no han desarrollado la capacidad de comprender globalmente lo que el niño quiere decir.

El niño intuitivamente a nivel analógico en la interacción con el adulto se da cuenta que lo que él quiso decir no fue plenamente escuchado o entendido por sus padres. Sin embargo la lectura explicita del padre o de la madre respecto a lo que el hijo dijo se constituye como "la verdad" a nivel consciente en el niño, debido a la omnipotencia que tienen las figuras de autoridad en la edad preescolar.

Entonces aprende a no creer en sus sensaciones e intuiciones ya que lo que le indica su sistema nervioso autónomo es contradictorio con el mensaje verbal explícito que está recibiendo. Aprende a desconfiar de sí mismo, de sus intuiciones y a no creer en sus sensaciones y emociones, especialmente si éstas indican una dirección distinta a la señalada como "adecuadas" por las figuras de autoridad con quienes mantiene un vínculo de dependencia emocional importante. Se convierte asi en "malo" y se avergüenza de sí mismo no sólo por su conducta, sino por el sólo hecho de ser un individuo que siente de una determinada forma que no es reconocida ni validada por el otro significativo.

\section{Diferenciación Personal: Un caminohaciaunaidentidad saludable}

Una persona que ha desarrollado una identidad con tendencia a la vergüenza puede en cualquier 
momento de su vida lograr integrar su identidad de un modo más sano y funcional. El camino es la diferenciación personal, facilitadaya sea por el curso de las experiencias de vida, la psicoeducación y/o la psicoterapia.

Tal como lo describe Erik Erikson, la adolescencia constituye el gran momento de la vida en el cual una persona puede integrar un mayor conocimiento de sí mismo puesto que antes cuando niño sólo tenia impresiones relativamente separadasy desconectadas acerca del self. Sin embargo no hay que olvidar que la estructuración de la identidad personal no termina en la adolescencia sino que se trata de un proceso que transcurre durante toda la vida.

De hecho, son muchas las crisis que viven las personas en distintos momentos de su ciclo vital. El concepto de crisis planteado por James Marcia (1979) en relación al desarrollo de la identidad en la adolescencia es aplicable para cualquier etapa de la vida. La entiende como el momento en el que la persona toma una decisión consciente y un compromiso ideológico y valórico en una determinada dirección. Cada una de las crisis de la vida son una oportunidad para que una persona se mire a si misma, vaya diferenciándose y revise su compromiso con sus valores y metas de vida albergando la posibilidad de reformular su identidad con un sentido más integrado de sí mismo.

Murray Bowen (1988) describe el concepto de "diferenciación del sí mismo", aludiendo a la capacidad de un individuo de tener conciencia si su funcionamiento es determinado emocional o intelectualmente en un determinado momento, lo cual le permite tener la libertad para elegir funcionar de un modo u otro en una situación dada. Por otro lado afirma que la diferenciación personal corresponde a la habilidad que tiene una persona para conservar su autonomía emocional dentro de los sistemas relacionales. Es decir, logra mantener los propios sentimientos, pensamientos y conductas al interior de relaciones emocionales cercanas sin fusionarse con el otro y perder su sí mismo, como también permitiéndole al otro ser el mismo.

La diferenciación personal, entendida entonces como un proceso de desarrollo del si mismo, de la autonomía e independencia emocional de los demás, y por ende del desarrollo de la autoconfianza y autoestima, permite al individuo ser activo en el mundo con una verdadera integración entre su sentir emocional y su hacer. Una persona diferenciada es capaz de legitimar sus sentimientos y conductas, permitiéndose equivocarse sin avergonzarse de cada emoción o conducta que difiera de las expectativas de los demás. También será capaz de comprender legitimamente el sentir y hacer de otra persona, por distinto que sea del suyo, lo que no implica necesariamente compartir sus ideas y/o sentimientos.

Una mayor diferenciación personal permite al individuo legitimar sus necesidades, vivir tranquilo su propia vida y no en función de los demás y aceptar el flujo sorpresivo de la vida. Le permite vivir en un único presente que conlleva un pasado y un futuro integrado, sin vivir en la culpa del pasado ni en la vergüenza de las expectativas presentes o futuras que tal vez no pueda satisfacer.

A una persona diferenciada le será más fácil desprenderse de la necesidad de mantener el control sobre su entorno. El control sobre las relaciones interpersonales y sobre los eventos que ocurren en la vida, constituye más bien una fantasía del que la experimenta, que un hecho en sí. Vivir en el control no permite experimentar la danza emocional espontánea que ocurre cuando dos o más individuos interactúan entre si (Maturana, 1993). En la medida que las relaciones interpersonales no estén basadas en el control, ni en las expectativas y/o exigencias mutuas, ni en función de los deberes sociales, habrá libertad, respeto por sí mismo y por el otro, satisfacción de las propias necesidades, empatía con las de los demás, autonomía e iniciativa y no vergüenza o culpa.

Rogers afirma que una persona plena y saludable es aquella que ha alcanzado un cierto nivel de congruencia entre lo que desea ser y lo que percibe que es (su yo ideal y su yo real). Es una persona capaz de aceptarse, ser ella misma y vivir sin mayores conflictos internos ni ansiedades. Destaca la necesidad de las personas de un afecto positivo incondicional por su valor y dignidad como seres 
humanos, independientemente de las conductas evaluadas socialmente aceptables o no.

Para que una persona desarrolle una identidad saludable es indispensable en sus relaciones familiares tempranas y posteriores relaciones interpersonales se produzcan actitudes de aceptación, empatía y autenticidad. Si este tipo de experiencias no han sido suficientemente frecuentes en la vida como para lograr una diferenciación del sí mismo que permita vivir fuera de la vergüenza, el proceso psicoterapéutico es un espacio que permite a una persona integrar una identidad coherente con sus auténticas necesidades. Este proceso es facilitado en este contexto puesto que alli se establece un clima de aceptación positiva incondicional que permite al consultante explorar sus sentimientos y experiencias que han sido negadas o distorsionadas anteriormente, logrando integrar su identidad de un modo saludable.

\section{Referencias}

Bukatko, D.; Daehler, M. (1995) Child Development. Houghton Mifflin Company. U.S.A.

Engler, B. (1996) Introducción a lasteorias delapersonalidad. Editorial Mc. Graw-Hill Mexico,

Erikson, E. (1974) Identidad, Juventud y Crisis. Editorial Paidós. Buenos Aires.

Harper, J .; Hoopes, M. (1990) Uncovering Shame: an approach integrating individuals and their family systems. W.W. Norton \& Company, New York.

Kerr, M. \& Bowen, M. (1988) Family Evaluation. Editorial W.W. Norton \& Company Inc. U.S.A.

Lewis, M. (1990) Child and adolescentpsychiatry. Editorial Williams \& Wilkins.

Maturana, H. \& Verden-Zöller, G. (1993) Amor y Juego: Fundamentos Olvidados de lo Humano. Editorial Instituto de Terapia Cognitiva. Santiago.

Papalia, D. ; Wendkos, S. (1997) Psicología del desarrollo. (6ºd) Editorial Mc. Graw Hill. Mexico.

Rice, P. (1997) Desarrollo humano:estudio del ciclo vital. Ed PrenticeHall Hispanoamericana, U.S.A.
Seifert, K.; Hoffnung, R. (1994) Child and Adolescent Development Houghton Mifflin Company. U.S.A.

Sepúlvecta, G. (1996) Identidad Personal en la Edad Juvenil. Consideraciones en relación a la filosofia politica de Hannah Arendt. Boletin Sociedad de Psiquiatria y Neurologia de la Infancia y la Adolescencia.

Watzlawick, P. Et al (1989) Teoria de la Comunicación Humana. Editorial Herder. Barcelona. 